\title{
Der Einfluß von Temperatur, Futter, Größe und Herkunft auf die sexuelle Differenzierung von Glasaalen (Anguilla anguilla)
}

\author{
H. KuHLMANN \\ Institut für Hydrobiologie und Fischereiwisscnschaft der Universität Hamburg; \\ Hamburg 50, Bundesrepublik Deutschland
}

\begin{abstract}
The influence of temperature, food, size and origin on the sexual differentiation of elvers (Anguilla anguilla). The effects of rearing and feeding conditions, as well as of body size and origin on sexual differentiation have been examined in elvers collected on the Atlantik coast (River Ems) and in the Tyrrhenian Sea. Raised at $17^{\circ}, 20^{\circ}, 23^{\circ}, 26^{\circ}$ or $29^{\circ} \mathrm{C}$ and at constant photoperiod, the elvers received either commercial fish food or cod roe. Eels from the Tyrrhenian Sea mostly developed into males. To some extent, at temperatures optimal for growth $\left(26^{\circ} \mathrm{C}\right)$ and feeding a cod-roe diet, a shift of sex ratio in favour of females was observed in Atlantic eels. The results obtained contradict those of Frdora (1951), who, rearing elvers in ponds, found the percentage of male eels to be positively correlated with increasing stock density. Although in the present study, stock density was 200 times higher than in FIDORA's experiments, the percentage of females was much higher than that of males. The results obtained support BELLINI's (1907) findings that big elvers preferentially develop into females, but small elvers into males. Irrespective of elvers' origin, water temperature and diet, the onset of sexual differentiation dependends upon attaining a certain body length $(15-25 \mathrm{~cm})$, but not on age. Whereas under natural conditions females are supposed to grow faster than males, under the conditions tested the males grew faster than the females. It is concluded, that, in addition to genetic factors, various environmental factors may influence sex determination in eels.
\end{abstract}

\section{EINLEITUNG}

Untersuchungen zur Geschlechtsdifferenzierung und zur Geschlechterverteilung des Aales wurden bisher fast ausschließlich an natürlichen Beständen durchgeführt (D’Ancona, 1959; Hornyold, 1931; Sinha \& Jones, 1967; Sivertsen, 1938; J. J. Tesch, 1928; Penaz \& F.-W. Tesch, 1970). Auf Grund der Ergebnisse dieser und vieler anderer Untersuchungen herrscht die Meinung vor, daß im Unterlauf der Flüsse und in den brackigen Mündungen vorwiegend männliche, im Oberlauf hingegen vorwiegend weibliche Aale anzutreffen sind. Viele Befunde stehen hierzu jedoch im Widerspruch: J. J. TESCH (1928) fand $94 \%$ O $\hat{\sigma}$ in der Zuidersee, hingegen nur $70 \%$ $\hat{\partial} \hat{\delta}$ in der weiter seewärts gelegenen Waddensee. Hornyold (1931) fing ô $\delta \hat{~ h i n-~}$ gegen weit entfernt vom Meer und $\not \circ$ im Salzwasser. SivertSEn (1938) beobachtete 
an der norwegischen Küste ein Geschlechterverhältnis von 9 of $\delta$ zu 665 oq. PeNAZ \& F.-W. Tesch (1970) fanden im Gegensatz zu allen bisherigen Befunden um Helgoland einen fast reinen Bestand an weiblichen Gelbaalen, während im inneren ElbeAstuar nahezu 90\% $\delta$ sowie ein hoher Anteil an unbestimmbaren Aalen und im Oberlauf fast nur Weibchen anzutreffen waren. Erstaunliche Unterschiede im Geschlechterverhältnis der Aale fand D'ANcona (1958). Nach seinen Untersuchungen besteht der Aalbestand des Tyrrhenischen Meeres zu 80\% aus Männchen, der der Adria hingegen zu fast $90 \%$ aus Weibchen.

Die Ergebnisse deuten darauf hin, daß mehrere Ursachen für die Unterschiede in der Geschlechterverteilung der Aalbestände an verschiedenen Fangplätzen in Betracht gezogen werden müssen. Die beiden heute vorwiegend vertretenen Theorien besagen, daß entweder die genetische Fixierung des Geschlechtes - zumindest beim größten Teil der Glasaale - labil ist und verschiedene Umwelteinflüsse das Verhälnis der Geschlechter in die eine oder andere Richtung verschieben können oder aber, daß Glasaale sexuell prädeterminiert sind und entsprechend ihrem Geschlecht ein unterschiedliches Wanderverhalten zeigen. Unter der Annahme, daß weiblich determinierte Aale einen stärkeren Wandertrieb haben, wäre es erklärlich, daß in den Oberläufen der Flüsse und am Rande des Verbreitungsgebietes (Adria, Ostsee, norwegische Küste) ein höherer Prozentsatz an weiblichen Aalen vorkommt (D’AnconA, 1957; 1958).

Die Hypothese der labilen genetischen Fixierung des Geschlechtes verdient besondere Beachtung. Hierzu liegen neben Beobachtungen an natürlichen Beständen auch einige Experimente mit gezielter Fragestellung vor. J. J. TEscH (1928) und GANDOLFI/HORNYOLD (1932) berichteten, daß es ihnen gelungen sei, durch besonders gutes Futterangebot bei Satzaalen aus einem Gebiet mit vorwiegend männlichen Aalen im Aquarium den Anteil an weiblichen Aalen zu erhöhen. Penaz \& F.-.W. Tesch (1970) beobachteten an natürlichen Beständen, daß ein gutes Wachstum in den Längenbereichen unter $30 \mathrm{~cm}$ mit einem hohen Weibchenanteil einherging. Fidora (1951) fand, daß bei der Teichaufzucht von Aalen mit zunehmender Besatzdichte der Anteil der männlichen Aale ansteigt. Der Einfluß der Wassertemperatur auf die sexuelle Differenzierung der Glasaale wurde von D'ANCoNA (1957) untersucht. Er kam zu dem Ergebnis, daß der Anteil der männlichen Aale mit steigender Wassertemperatur zunimmt. Mazza (1932) berichtete von einer Geschlechtsumwandlung bei einem männlichen Blankaal, der in Gefangenschaft gehalten wurde, die sich ohne hormonelle Behandlung vollzog. Er führte dazu an demselben Tier mehrfach eine Biopsie durch.

Neben diesen Untersuchungen wurden eine Anzahl von Transplantationsversuchen durchgeführt (GANdolfi/Hornyold, 1931, 1932; Frost, 1950; Bertrn, 1951; D'Ancona, 1959), die zum Ziel hatten, Aale aus Gebieten, in denen vorwiegend das eine Geschlecht vertreten war, durch Transplantation in Gebiete, in denen vorwiegend das andere Geschlecht vertreten war, zur Geschlechtsumkehr zu veranlassen. Die Ergebnisse dieser Experimente fielen allerdings widersprüchlich aus. In den meisten Fällen jedoch ist der Aussagewert dieser Versuche wegen der hohen Mortalität, nicht konstanten Versuchsbedingungen, Fehlen histologischer Kontrollen und unvollkommener Versuchsbeschreibungen begrenzt bzw. statistisch nicht abzusichern. 


\section{MATERIAL UND METHODE}

Als Besatzmaterial für die hier beschriebenen Experimente wurden unpigmentierte Glasaale (Anguilla anguilla) aus dem Atlantik (Herbrum/Ems) und aus dem Mittelmeer (Tyrrhenisches Meer) verwendet. $\mathrm{Da}$ aus der Literatur bekannt ist, daß die Größe der Glasaale vom Zeitpunkt des Fanges abhängt (Heldr \& Heldr, 1929; Matsui, 1952), wurde darauf geachtet, daß beide Fänge etwa in der Mitte der Fangsaison lagen. Auf diese Frage wurde Wert gelegt, da das Wachstum und damit möglicherweise auch die Geschlechtsdifferenzierung der Glasaale von ihrer Länge abhängt (Matsut, 1952). Dennoch waren die Mittelmeeraale mit einer mittleren Länge von $6,4 \mathrm{~cm}$ wesentlich kleiner als die atlantischen Aale mit einer mittleren Länge von $7,5 \mathrm{~cm}$.

Die Aufzucht der Aale erfolgte in einem Warmwasser-Kreislaufsystem mit Kiesfilter. Dem System wurden täglich etwa $20 \%$ Frischwasser zugesetzt. Die Wasserqualität war mit den folgenden über die gesamte Versuchsdauer gemittelten Werten als sehr gut zu bezeichnen: $\mathrm{pH}-7,2 ; \mathrm{NH}_{4}-0 ; \mathrm{NO}_{2}{ }^{-}-0,1 ; \mathrm{NO}_{3}{ }^{-}-4,0 \mathrm{mg} / \mathrm{l} ; \mathrm{O}_{2}-$ Sättigung $95 \%$.

In den 200-1-Aufzuchtbecken wurden mit einer Schwankung von $\pm 0,5^{\circ} \mathrm{C}$ Temperaturstufen von $17^{\circ}, 20^{\circ}, 23^{\circ}, 26^{\circ}$ und $29^{\circ} \mathrm{C}$ eingestellt; der Durchfluß betrug etwa $5 \mathrm{l} / \mathrm{min}$. Uber jedem Becken war eine Leuchtstoffröhre angebracht. Die Beleuchtung war konstant von 7.00-20.00 h eingeschaltet (LD 13:11).

Gefüttert wurde mit einem Futterbrei aus Forellenfertigfutter bzw. KabeljauRogen mehrmals täglich bis zur Sättigung aller Aale (weitere Angaben in KuHLMANN, 1974).

Jedes Versuchsaquarium (Grundfläche $0,5 \mathrm{~m}^{2}$ ) wurde mit 200 Glasaalen beset $z$ t. Die Bestände aller Aquarien hatten die gleiche Durchschnittslänge und die gleiche Längenverteilung. Jeder einzelne Versuchsbestand wurde durch Längensortierung in 2 Untergruppen von je 100 Aalen unterteilt, wobei die eine Gruppe aus Aalen $<\bar{x} L$ und die andere aus Aalen $>\bar{x} L$ des Gesamtbestandes bestand. Die beiden so gebildeten Gruppen wurden in demselben Aquarium, getrennt durch eine Gazewand, aufgezogen. Die beiden Aquarienhälften waren mit separatem Wasserzu- und -abfluß sowie Belüftung versehen. Die Aufteilung der Versuchsbestände sollte u. a. dazu dienen, eventuelle Unterschiede in der sexuellen Differenzierung großer und kleiner Aale beobachten zu können und die Verluste durch Kannibalismus zu verringern.

Nach einer Aufzuchtdauer von etwa 11/4 Jahren wurden alle Aale zur histologischen Überprïfung der Gonadendifferenzierung getötet, in Salpetersäure entkalkt, in einer modifizierten Lösung nach Davidson (RomeIs, 1968) konserviert und in Paraplast eingebettet (vgl. Kunlmann, 1974). Gefärbt wurde mit Hämalaun nach Meyer ohne Gegenfärbung oder mit Azan nach Heidenhain (Romers, 1968).

Zur Interpretation der Gonadendifferenzierung wurden Schnitte von $7 \mu \mathrm{m}$ Dicke aus der "zentralen Zone ${ }^{*}$ der Gonaden angefertigt, in der nach RoDolico (1933) die Differenzierung der Gonaden beginnen soll. 


\section{ENTWICKLUNG DER GONADEN}

Die paarigen Gonaden des Aales liegen im dorsalen Bereich der Leibeshöhle zu beiden Seiten der Wirbelsäule unterhalb der Nieren (Abb. 1). Je nach Reifegrad des Aales sind sie als winzige Peritonealfalte oder als mehr oder weniger massives Organ ausgebildet (Abb. 2).

Einige Aale $(>25 \mathrm{~cm}$ Länge) konnten - unabhängig von der späteren histologischen Kontrolle - bereits makroskopisch nach Ơffnen der Leibeshöhle eindeutig als Männchen oder Weibchen identifiziert werden.

Bei frisch getöteten Aalen von etwa $20-25 \mathrm{~cm}$ Länge war es in vielen Fällen möglich, das männliche Geschlecht dadurch nachzuweisen, daß die Gonade an ihrem cra-

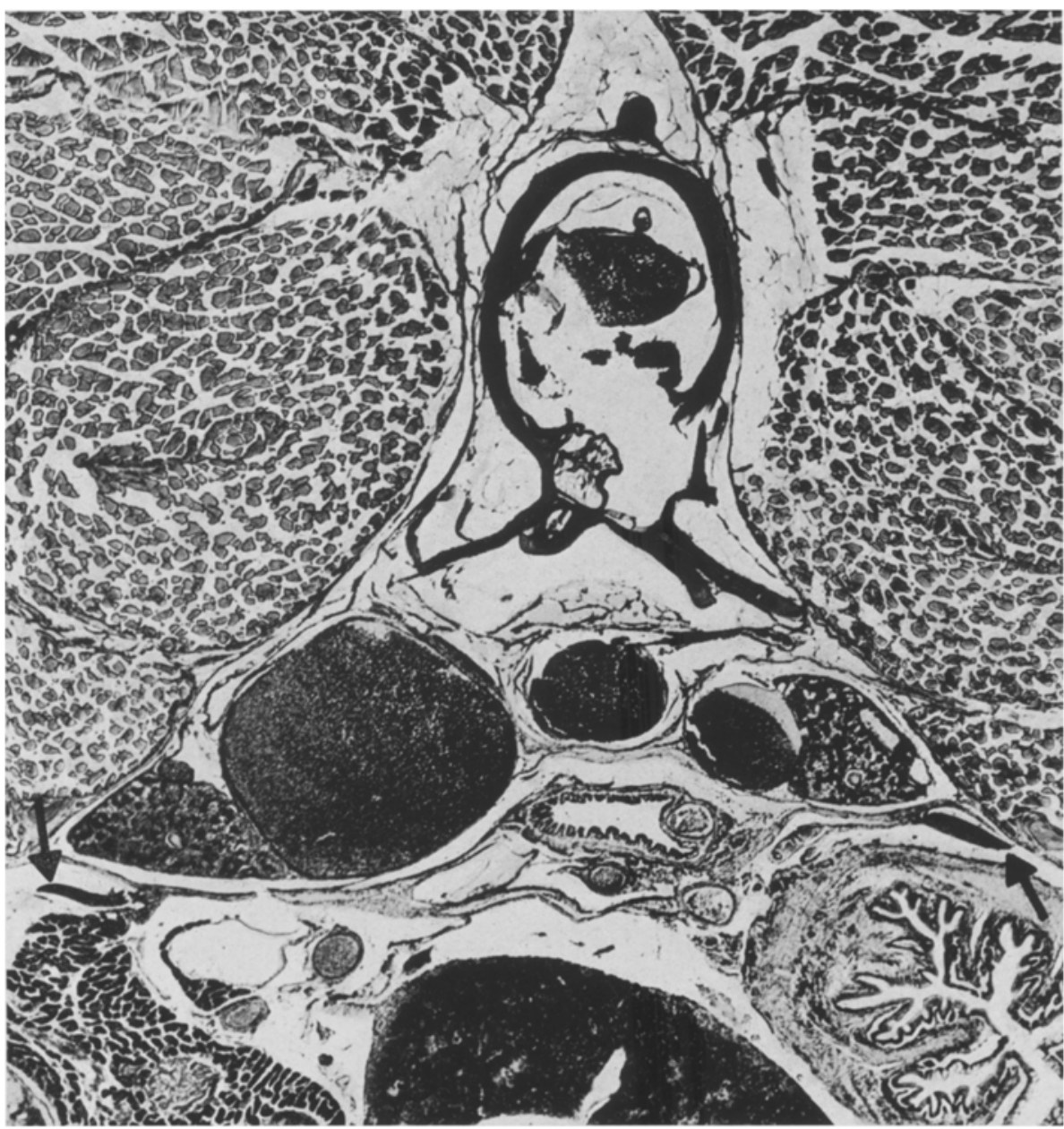

Abb. 1: Querschnitt durch einen Aal von $21 \mathrm{~cm}$ Länge. Die Pfeile markieren die Lage der paarigen Gonaden $(9,5: 1)$ 
nialen Teil mit der Pinzette gefaßt und als glasklarer Faden mit tröpfchenförmigen einseitigen Verdickungen (Loben) in caudaler Richtung fast in ihrer gesamten Länge herausgezogen werden konnte. Eine spätere histologische Uberprüfung der so gewonnenen Präparate ergab in jedem Fall eine Identifizierung des männlichen Geschlechts.

War die Gonade nicht in einem Stück herauszuziehen - vermutlich infolge Feh-

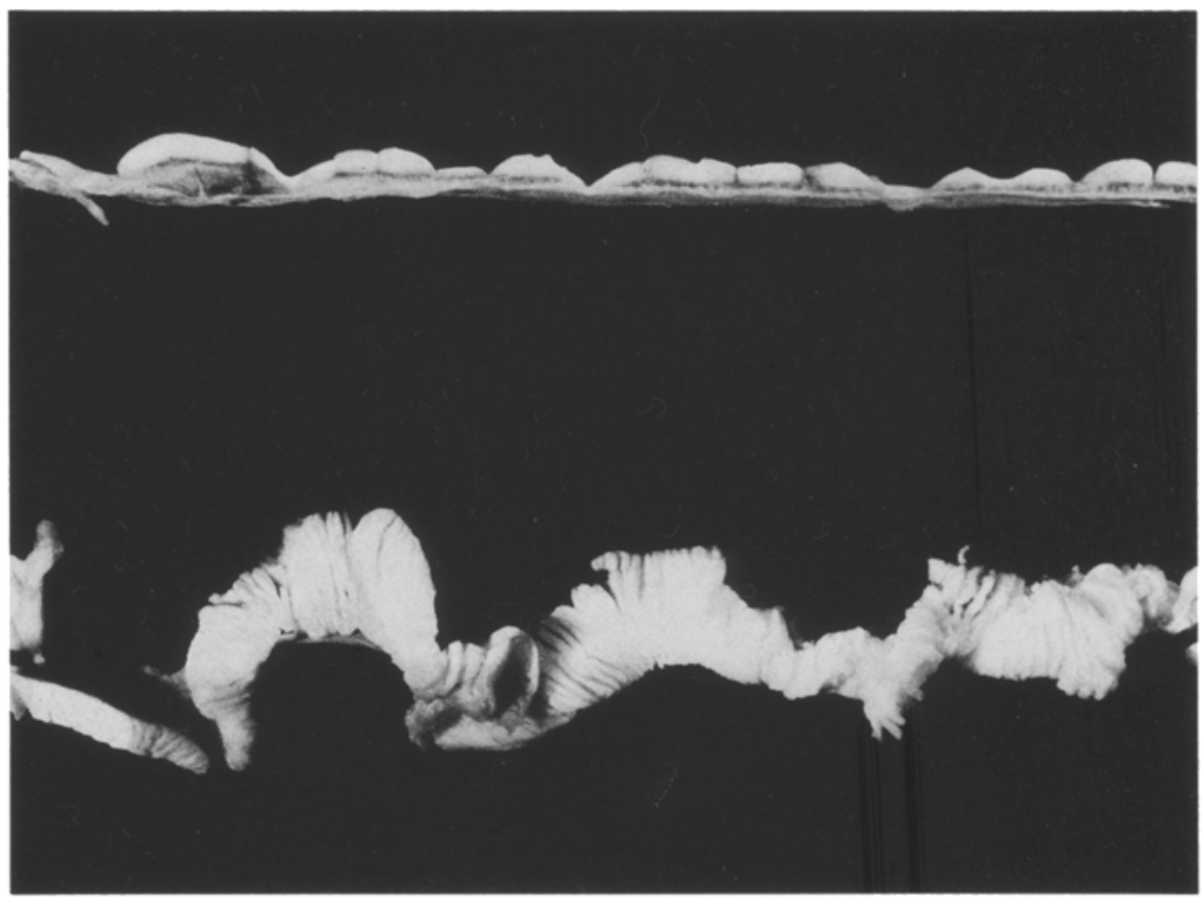

Abb. 2: Totalpräparat der männlichen Gonade eines Aales von $42 \mathrm{~cm}$ Länge (oben) und der weiblichen Gonade (unten) eines Aales von $57,5 \mathrm{~cm}$ Länge (2:1)

lens des recht massiven Vas deferens - so handelte es sich um wenig differenzierte Weibchen, kaum differenzierte Männchen oder undifferenzierte Aale. Bei Aalen unter $20 \mathrm{~cm}$ Länge war die Gonade nur durch Totalschnitte der Tiere zu erkennen.

Für die Interpretation der Schnitte stehen bisher auch in der Literatur keine zuverlässigen statistischen Angaben zur Verfügung. Protogonienzählungen (RoDolico, 1933) führten zu keinem Ergebnis. Die Interpretation der Schnitte erfolgte daher unter Verwendung der in der Literatur insbesondere von RoDol.rco (1933) und D'ANCONA (1943, 1957) angegebenen Kriterien. Im folgenden soll die Entwicklung der Aalgonade von der undifferenzierten winzigen Genitalleiste bis zum eindeutig differenzierten Aal kurz umrissen und dabei die entscheidenden Kriterien für die einzelnen Entwicklungsstadien herausgestellt werden. 


\section{Erste Gonadenanlage bei Glasaalen und kleinen Steigaalen}

In einer kleinen, im Querschnitt keulenförmigen Genitalleiste, die von peritonealem Epithel umgeben ist, finden sich etwa 2-6 Urgeschlechtszellen, für die im folgenden die Bezeichnung "Protogonien“ nach Beccari (1932) gilt. Die Protogonien besitzen sehr viel helles Cytoplasma und sind daher etwa 2-3mal größer als die somatischen Zellen. Protogonien in Teilung sind nie anzutreffen (Rodolico, 1933). Histologisch sind keinerlei sexuelle Differenzierungen zu erkennen.

\section{Wachstum der Gonade durch Vermehrung der Protogonien}

Durch die beginnende Teilung der Protogonien nimmt die Gonade an Umfang zu; die einzelnen Protogonien werden allerdings durch die Teilungen etwas kleiner (Deutogonien). Das histologische Bild der Gonade ändert sich in der Struktur nicht wesentlich, es ist noch keine sexuelle Differenzierung der Gonade zu erkennen. Nestartige Zellanhäufungen sind noch nicht $z u$ finden.

\section{Jugendliche Oogenese}

Nach Rodolico (1933) vollzieht sich dieser Vorgang bei allen Aalen. Es entstehen unregelmäßig verteilte Nester von etwa 8-16 Zellen (Oogonien), ohne daß damit eine endgültige Feminisierung der Gonade eingeleitet wird. Es soll sich hierbei um ein protogynes $Z$ wischenstadium der Gonadenentwicklung handeln.

\section{Intersexuelles Stadium}

In diesem Stadium sind sowohl Spermatogonien, als auch Oogonien (Nester) normal ausgebildet und existieren meist unregelmäßig verteilt nebeneinander. Die Oogonien sind weiterhin durch ihre Ansammlung in Nestern zu erkennen, vereinzelt treten allerdings auch schon isolierte Oocyten auf. Die Spermatogonien sind z. T. noch als kleinere isolierte Zellen zu erkennen, treten allerdings häufiger auch schon in der ganz charakteristischen "Kettenform" auf, einer Struktur, die der Ausbildung der späteren Tubuli seminiferi vorausgeht. Als weiteres typisches Zeichen maskuliner Elemente in der Gonade gilt das Auftreten interstitieller Zellen, die bevorzugt die Spermatogonienketten voneinander isolieren. In diesem Stadium sind zahlreiche Zellteilungen zu beobachten.

\section{Beginnende sexuelle Differenzierung und fortschreitende Degeneration der männlichen bzw. weiblichen Geschlechtszellen}

Nach Grassi (1919), Rodolico (1933) und anderen Autoren setzt die eigentliche sexuelle Differenzierung der Aalgonade durch Rückbildung des einen oder anderen 
Geschlechtszellentyps ein. Beide Autoren glauben, daß diese Degeneration von Umweltbedingungen abhängig ist und so das unterschiedliche Geschlechterverhältnis an verschiedenen Orten entstehen könne.

\section{Differenzierung und Weiterentwicklung der weiblichen Gonade}

Wie Bellini (1907), Rodolico (1933) und andere Autoren annehmen, entwickeln sich etwa $10 \%$ aller Glasaale unter Überspringen des intersexuellen Stadiums direkt zu Weibchen. Sie sollen in ihrer sexuellen Differenzierung von der Umwelt nicht beeinflußt werden. Die meisten Aale durchlaufen jedoch ein Stadium der Intersexuali-

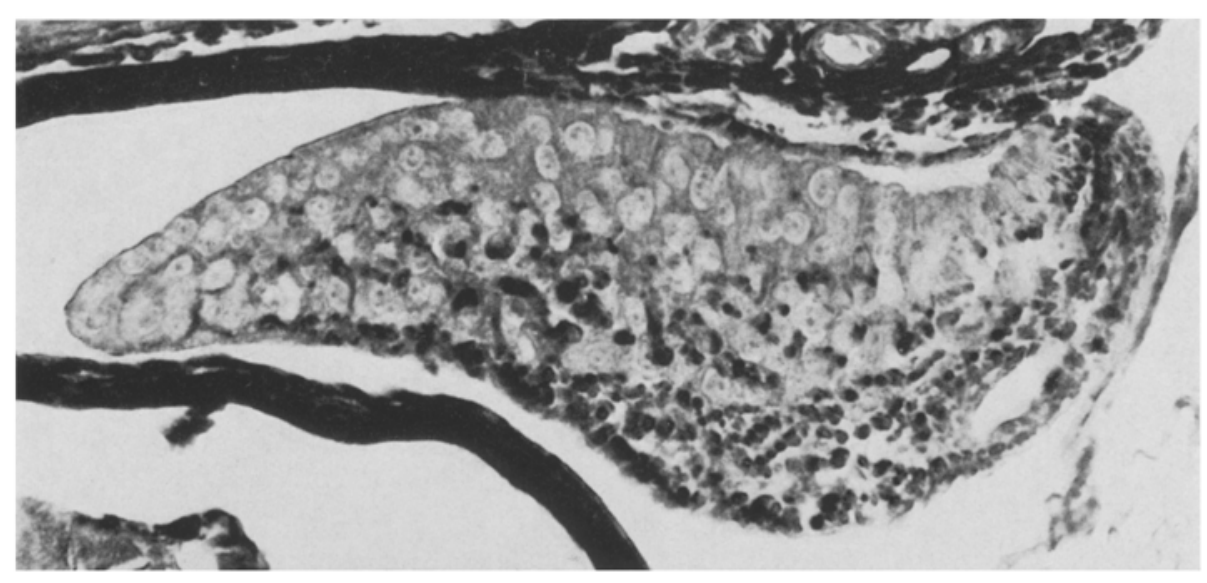

Abb. 3: Querschnitt durch die Gonade eines Aales („frühes Weibchen“) von 19,2 cm Länge (ca. $400: 1)$

tät. Im Verlaufe dieser Entwicklung gewinnt in der später weiblichen Gonade die Produktion von Oogonien und Oocyten die Oberhand. Scheinbar unabhängig davon geht die Produktion von normalen Spermatogonien - allerdings verlangsamt - weiter. Anhäufungen von nicht degenerierten Spermatogonien in der Prophase können selbst in vielen eindeutig voll ausdifferenzierten weiblichen Gonaden in sehr geringer Zahl zunächst noch angetroffen werden.

Unter den konstant gehaltenen Bedingungen der hier beschriebenen Experimente wurde ein starkes zahlenmäßiges Uberwiegen der Oogoniennester oder der einzelnen Oocyten als Indiz für die beginnende endgültige Feminisierung („frühes Weibchen“) gewertet. Wie auch bei Aalen aus natürlichen Gewässern ist die Degeneration oder das völlige Verschwinden der Spermatogonien ein weiteres entscheidendes Kriterium für die endgültige Feminisierung der Gonade.

Im Verlaufe der weiteren Entwicklung erfolgt eine starke Vergrößerung der Gonade dadurch, daß neue Nester von Oogonien entstehen, und die alten Nester in 


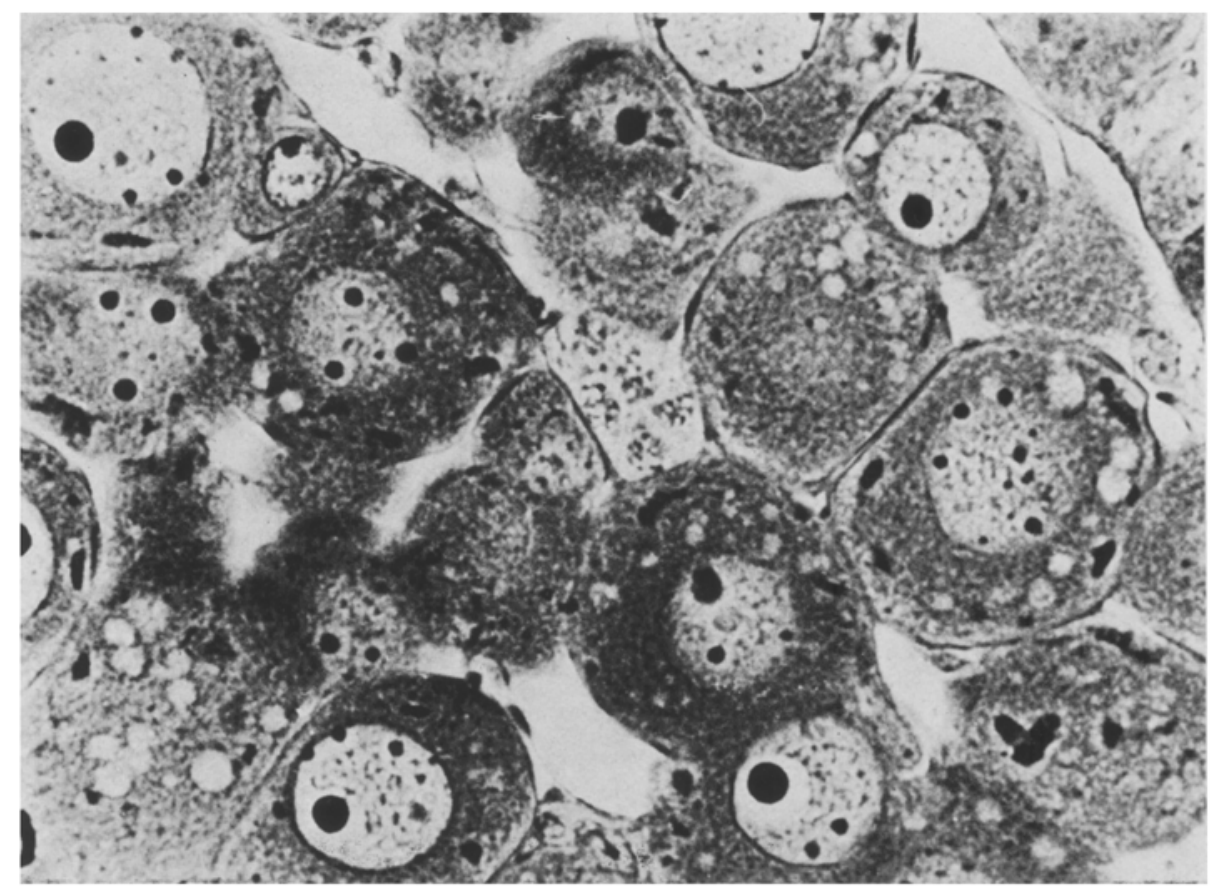

Abb. 4: Querschnitt durch die Gonade eines weiblichen Aales von $28,7 \mathrm{~cm}$ Länge. Die Gonade ist erfüllt mit Oocyten, die Reservematerial (Oltröpfchen) eingelagert haben und von Follikelzellen umgeben sind (ca. $625: 1$ )

einzelne, wachsende Oocyten zerfallen, bis schließlich die ganze Gonade von einzelnen Oocyten erfüllt ist und keine Nester mehr anzutreffen sind (Abb. 3).

Die weitere Vergrößerung der Gonade erfolgt nun fast ausschließlich durch das Wachstum der von mehreren Follikelzellen umgebenen Oocyten, die Reservematerial in Form von Olkugeln einlagern (Abb. 4). Dabei wird die Struktur der Gonade etwas aufgelockert und die äußere Form immer unregelmäßiger entsprechend ihrer späteren Form als sogenanntes „Krausenorgan“ (Abb. 2).

\section{Differenzierung und Weiterentwicklung der männlichen Gonade}

Analog zur weiblichen Gonade ist die Degeneration der Oocyten ein deutliches Zeichen der Maskulinisierung der Gonade. Im histologischen Bild ist die Degeneration durch den tiefgefärbten pyknotischen Kern der Oocyten zu erkennen. Sie kann schon auf dem Stadium der Oogonien-Nestbildung beobachtet werden, tritt jedoch in den meisten Fällen erst bei größeren wachsenden Oocyten häufiger ein. In seltenen Fällen kann eine Degeneration der Oocyten auch noch sehr spät in völlig ausdifferenzierten weiblichen Gonaden beobachtet werden.

Andererseits können sich nicht degenerierte Oocyten noch sehr lange in völlig aus- 
differenzierten männlichen Gonaden halten und erst auf einem späten Differenzierungsstadium der Gonade degenerieren (Abb. 5).

Neben der bereits erwähnten von intensiv angefärbten interstitiellen Zellen begleiteten kettenförmigen Anordnung der Spermatogonien kann dic Ausbildung des Vas deferens in manchen Fällen als Zeichen der Maskulinisierung gewertet werden. Da

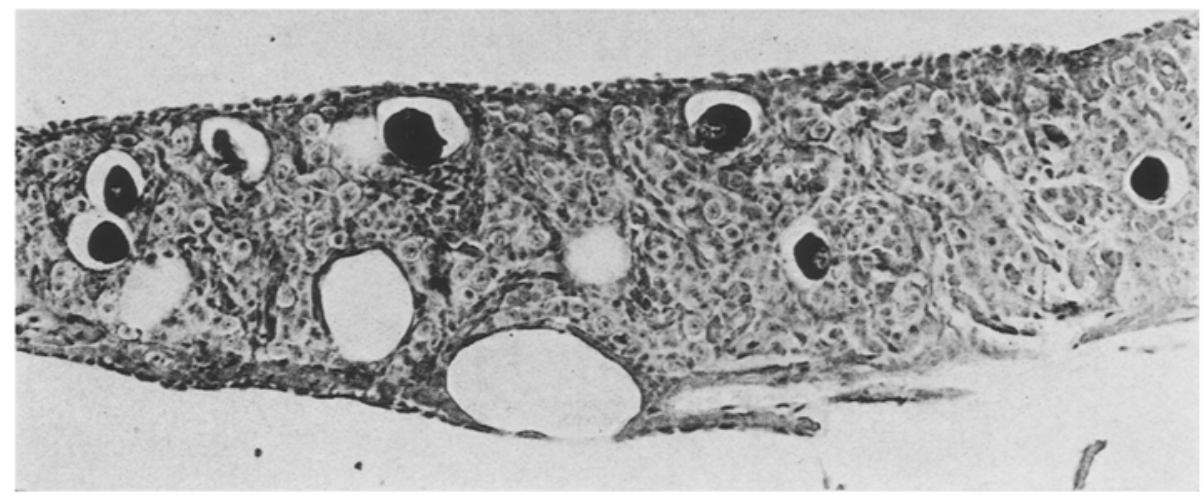

Abb. 5: Querschnitt durch die voll differenzierte Gonade eines männlichen Aales von $34,9 \mathrm{~cm}$ Länge. In der Gonade finden sich zahlreiche degenerierte Oocyten (ca. 230:1)

das Vas deferens bei noch schwach ausgebildeter Kettenstruktur schon sehr deutlich vorhanden sein kann, andererseits bei ausgeprägter Kettenstruktur kaum zu erkennen ist oder ganz fehlt, sollte das Vas deferens also nur in Verbindung mit dem übrigen histologischen Bild der Gonade gewertet werden.

Im Verlaufe der weiteren Entwicklung nimmt die Zahl der Spermatogonien und der Spermatocyten ständig zu, wodurch die Gonade vergrößert und die Kettenstruktur immer deutlicher ausgebildet wird. Das Vas deferens ist dann in jedem Fall deutlich ausgebildet. Während die „Tubuli" zunächst noch Spermatogonien und Spermatocyten enthalten, findet man auf späteren Stadien neben Spermatocyten auch schon sehr reichlich Spermatiden. Die Lobatur der Gonade ist auf diesem Stadium schon recht scharf ausgeprägt, und man kann mit völliger Sicherheit eine makroskopische Bestimmung des Gonadengeschlechtes vornehmen (Abb. 2).

\section{ABLAUF DER SEXUELLEN DIFFERENZIERUNG IN DEN VERSUCHSGRUPPEN}

Insgesamt wurden 427 Aale geschnitten und histologisch ausgewertet. Der Längenbereich dieser Aale erstreckte sich von 9-60 cm. Von jedem Aal wurden etwa 10-50 Schnitte angefertigt, die einen Bereich von 2-4 mm der zentralen Gonadenzone erfaßten. Innerhalb dieses Bereiches war das histologische Bild für die einzelne Gonade weitgehend einheitlich. Gonaden mit etwa gleicher Anzahl beider Geschlechtszellen wurden als „intersexuell“, Gonaden mit überwiegend Spermatocyten bzw. Oocyten als „frühes 
Stadium" (Männchen oder Weibchen) eingeordnet. Die Interpretation der Präparate wurde mehrfach in größeren Zeitabständen wiederholt. Für das Gesamtmaterial ergab sich dabei die in Tabelle 1 zusammengefaßte Aufteilung. Diese zeigt, daß bei den atlantischen Aalen am Ende der Versuche die Weibchen dominierten. Hingegen war bei den Aalen aus dem Mittelmeer die Zahl der Männchen wesentlich größer. Auf die Abweichungen bei der sexuellen Differenzierung der Mittelmeeraale wird an einer späteren Stelle eingegangen. Die Zahl der Intersexe ist bei den Mittelmeeraalen geringer als bei den atlantischen Individuen, da sie länger im Versuch waren und daher sich bereits ein größerer Prozentsatz sexuell differenziert hatte.

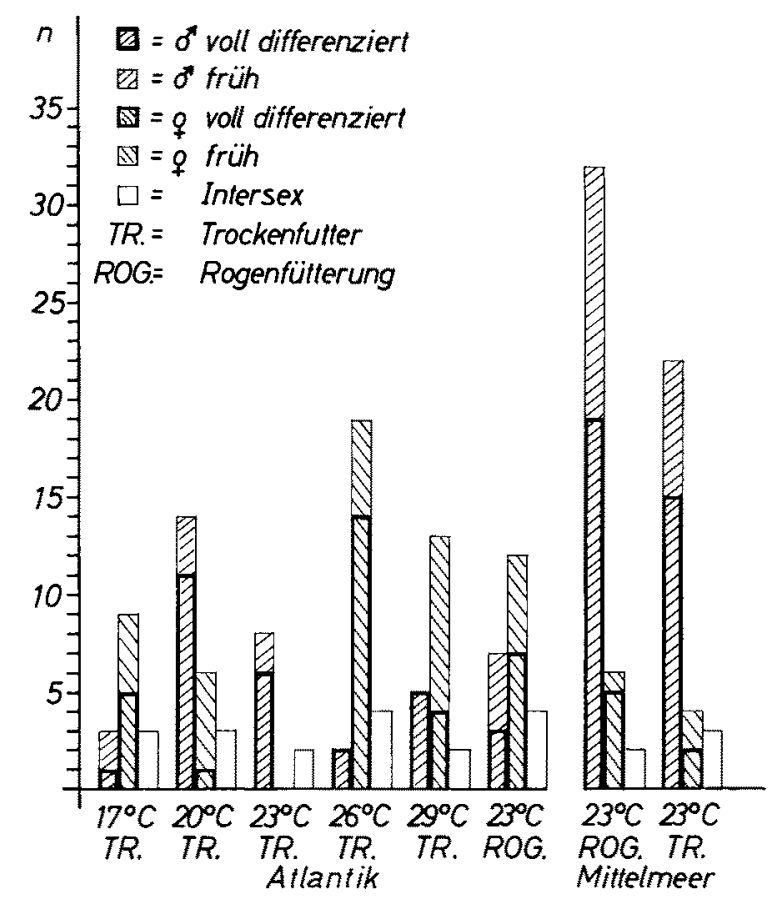

Abb. 6: Geschlechtsverhältnis aller sexuell differenzierten Aale in Abhängigkeit von der geographischen Herkunt sowie verschiedenen Temperatur- und Fütterungsbedingungen

In Abbildung 6 ist die Häufigkeit der verschiedenen Differenzierungsstadien für das gesamte Untersuchungsmaterial zusammengefaßt. Es wird deutlich, daß durch die Hinzunahme der "frühen Stadien" das Ergebnis in seiner grundsätzlichen Aussage nicht geändert wird.

Da für die „frühen Stadien“ die weitere Entwicklung der Gonaden nicht mit letzter Sicherheit vorausgesagt werden kann, sollen im folgenden für die Beurteilung der Ergebnisse ausschließlich solche Tiere herangezogen werden, bei denen eine eindeutige und endgültige Bestimmung des Geschlechtes durchgeführt werden konnte. 


\section{Die sexuelle Differenzierung der Aale von verschiedenen Fangplätzen}

Vergleicht man das Geschlechtsverhältnis der voll differenzierten Aale aus dem Atlantik mit dem der Aale aus dem Mittelmeer (Tab. 1), so ergibt sich ein Verhältnis von $19: 0,9$ für die atlantischen Aale und $1 q$ : 5,0 of fö die Aale aus dem

Tabelle 1

Geschlechterverhältnis aller sexuell differenzierten Aale

\begin{tabular}{|c|c|c|c|c|}
\hline \multirow{2}{*}{$\begin{array}{l}\text { Differenzierungsstadium } \\
\text { 우 voll differenziert } \\
\text { ợ früh differenziert }\end{array}$} & \multicolumn{2}{|c|}{$\begin{array}{c}\text { Atlantische Aale } \\
\text { n }\end{array}$} & \multicolumn{2}{|c|}{$\underset{\mathrm{n}}{\operatorname{Mittelmeeraale}} \underset{\%}{\mathrm{o}}$} \\
\hline & $\begin{array}{l}31 \\
28\end{array}$ & $\begin{array}{l}26,6 \\
24,2\end{array}$ & $\begin{array}{l}7 \\
3\end{array}$ & $\begin{array}{r}10,1 \\
4,3\end{array}$ \\
\hline Intersexuelles Stadium & 18 & 15,5 & 5 & 7,2 \\
\hline $\begin{array}{l}\delta \delta \text { früh differenziert } \\
\delta \delta \text { voll differenziert }\end{array}$ & $\begin{array}{l}11 \\
28\end{array}$ & $\begin{array}{r}9,5 \\
24,2\end{array}$ & $\begin{array}{l}20 \\
34\end{array}$ & $\begin{array}{l}29,0 \\
49,4\end{array}$ \\
\hline Undifferenziert & 377 & & 50 & \\
\hline
\end{tabular}

Mittelmeer. Dieses Resultat stimmt sehr gut mit der Angabe von D'ANCona (1958) über das Geschlechtsverhältnis der Aale aus dem Tyrrhenischen Meer uiberein.

\section{Die sexuelle Differenzierung unter dem Einfluß verschiedener Futtermittel}

Aus Abbildung 6 ist das Geschlechtsverhältnis der Aale nach Verfütterung von Forellenfertigfutter bzw. Kabeljau-Rogen zu ersehen. Das in den Experimenten gefundene Verhältnis der Geschlechter bei den atlantischen Aalen deutet darauf hin, daß die Verfütterung von Rogen eine Verschiebung der Geschlechtsverteilung zugunsten der Weibchen bewirkt. Diese Tendenz konnte jedoch bei den Experimenten mit den Aalen aus dem Mittelmeer nicht bestätigt werden. Allerdings stammen diese Aale aus dem Tyrrhenischen Meer, wo nach den Angaben von D'Ancona (1958) ohnehin 80\% aller Aale Männchen sind.

$\mathrm{Ob}$ im Falle der Rogenfütterung der höhere Nährstoffwert des Futters oder ein möglicher Gehalt an Geschlechtshormonen von Bedeutung war, ist ungewiß. Daß man beim Aal durch die Beimischung von weiblichen Geschlechtshormonen zum Futter den Anteil an Weibchen erhöhen kann, hat u. a. LIEDER (1969) gezeigt.

\section{Die sexuelle Differenzierung in verschiedenen Temperaturstufen}

Abbildung 6 zeigt ferner das Ergebnis der unterschiedlichen Temperaturbehandlung bezüglich der Geschlechtsentwicklung der Aale. Dabei wird deutlich, daß eine 
Korrelation zwischen Wassertemperatur und Geschlechterverhältnis der Aale für den gesamten untersuchten Temperaturbereich aus den durchgeführten Experimenten nicht hergeleitet werden kann, gleichgültig, ob man ausdifferenzierte und "frühe" Stadien gemeinsam oder beide Stadien für sich betrachtet.

Während bei $17^{\circ} \mathrm{C}$ ein $\delta \delta: q q-V e r h a ̈ l n i s$ von $1: 5$ auftritt, hat sich im Bereich von $20^{\circ} \mathrm{C}$ und $23^{\circ} \mathrm{C}$ der $\delta^{\circ} \delta$-Anteil stark erhöht (bei $20^{\circ} \mathrm{C}$ oै $\delta: q \%=11: 1$; bei $23^{\circ} \mathrm{C}$ ô $\delta$ : $+9=6: 0$ ). Bemerkenswert ist jedoch, daß im Bereich der für die meisten natürlichen Gewässer hohen Temperaturen von $26^{\circ} \mathrm{C}$ das Geschlechterver-

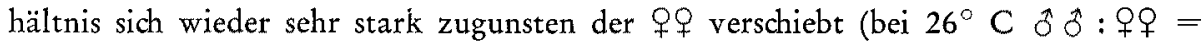
2 : 14). In diesem Zusammenhang sei erwähnt, daß bei den gleichzeitig durchgeführten Wachstumsuntersuchungen sich eine Wassertemperatur von $26^{\circ} \mathrm{C}$ als optimal herausgestellt hat. Ferner sei zu diesem Problem auf eine neuere Beobachtung aus der Praxis hingewiesen, daß Glasaale, die zunächst bei einer Wassertemperatur von $26^{\circ} \mathrm{C}$ vorgestreckt wurden, dann aber zur Mast in abgeschlossenen künstlichen Teichen gehalten wurden, bei der Abfischung einen 9 - - Anteil von $80 \%$ aufwiesen (mündliche Mitteilung von Fischzuchtmeister STÄHLER, Niederzeuzheim).

Der Rückgang des 우-Anteils bei $29^{\circ} \mathrm{C}$ mag darin begründet sein, daß mit dieser Temperatur das Optimum für die Aale bereits überschritten war, was durch eine Wachstumsdepression deutlich wurde. Ein Beweis, wonach hohe Wassertemperaturen das Geschlechterverhältnis der Aale zugunsten der ô ô verschieben (D'ANCoNA, 1957), scheint hiernach nicht gegeben.

\section{Körpergröße und sexuelle Differenzierung}

Für die Ermittlung des Längenbereiches, in dem die sexuelle Differenzierung der Aale beginnt, wurden die „frühen" Stadien unabhängig von dem zu erwartenden Geschlecht ausgewertet. Wie Abbildung 7 zeigt, begann in diesen Experimenten die sexuelle Differenzierung der Aale im Längenbereich von $15-25 \mathrm{~cm}$. Offensichtlich ist dieser Beginn unabhängig vom Alter, der Wassertemperatur, der Ernährung und der Herkunft der Aale; entscheidend ist lediglich das Erreichen eines bestimmten Längenbereiches. Auffallend ist, daß - bis auf eine Ausnahme - in allen Experimenten die mittlere Länge der "frühen Weibchen“ unter der der „frühen Männchen“ lag. Dieser Befund steht im Einklang mit der vielfach vertretenen Auffassung (D'ANCoNA, 1943; Rodolico, 1933; u. a.), daß die Aale in ihrer sexuellen Entwicklung ein protogynes Stadium durchlaufen sollen.

Ein Zusammenhang zwischen Durchschnittslänge der voll differenzierten Aale und der Wassertemperatur, der aus Abbildung 7 entnommen werden könnte, ist nur scheinbar. Er entspricht der Staffelung der Wachstumsleistung der einzelnen Versuchsbestände in Abhängigkeit von der Wassertemperatur.

Durch die oben beschriebene Aufteilung der Versuchsbestände konnte die sexuelle Differenzierung der Aale aus den Versuchshälften mit "kleinen" Glasaalen und die der Aale aus den Versuchshälften mit "großen“ Glasaalen getrennt ausgewertet werden. In Tabelle 2 ist für alle in dieser Hinsicht auswertbaren Versuche die Zahl der Männchen und Weibchen nach Versuchsgruppen getrennt aufgestellt worden. Es lassen sich fol- 


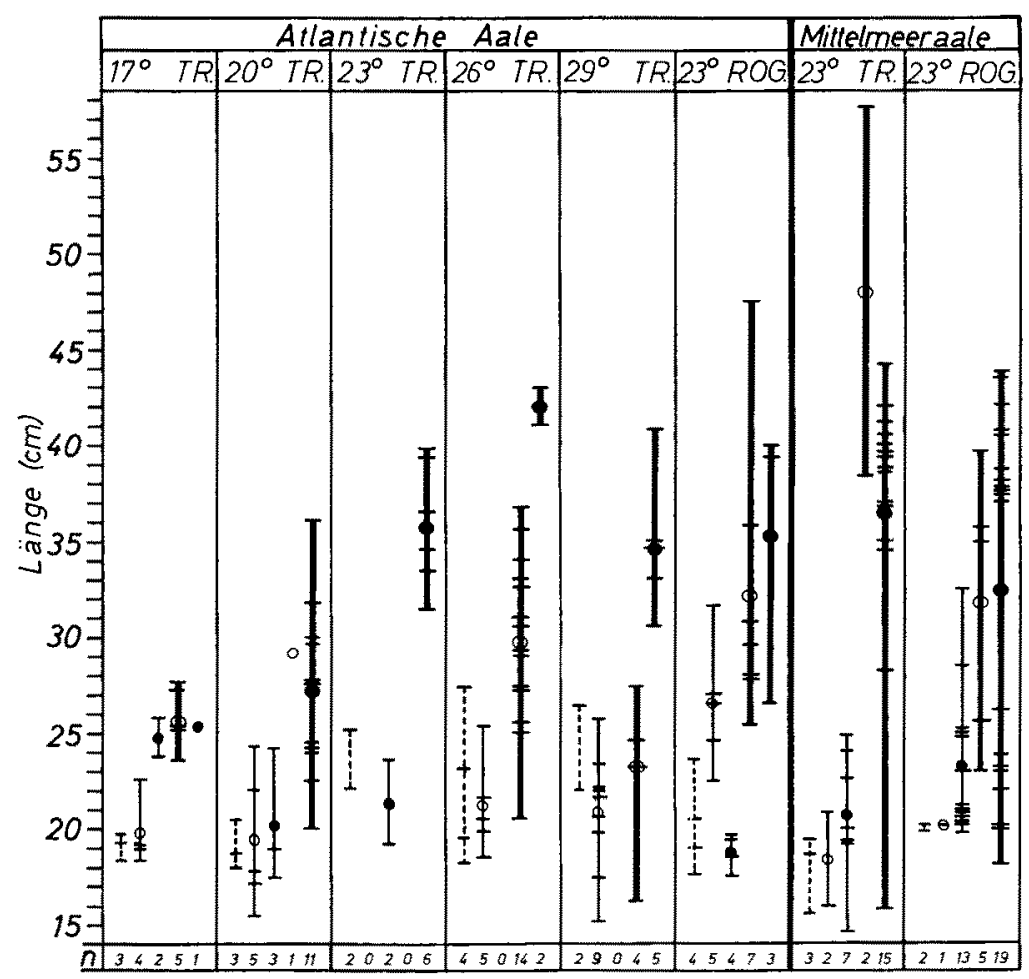

Intersex q früh $\Phi$ strüh qvoll diff. $\Phi$ ठ̀ voll diff. $\mathbf{I}$

Abb. 7: Mittlere Längenverteilung und Variationsbreite der verschiedenen Differenzierungsstadien in Abhängigkeit von der geographischen Herkuntt sowie verschiedenen Temperaturund Fütterungsbedingungen (TR. = Trockenfutter, ROG. = Rogenfutter)

gende Tendenzen erkennen: Bei den atlantischen Aalen waren in den Versuchshälften mit größeren Glasaalen am Ende der Experimente mehr Weibchen vorhanden, als in den Versuchshälften mit kleineren Glasaalen. Ein entsprechender Befund konnte für die Aale aus dem Tyrrhenischen Meer nicht in dem Maße bestätigt werden. Sowohl für die atlantischen als auch für die Aale aus dem Mittelmeer gilt jedoch die Tatsache, daß die Zahl der sexuell differenzierten Aale in den Versuchshälften, die mit größeren Glasaalen begonnen worden waren, z. T. erheblich größer war als in den Versuchshälften, die mit kleineren Glasaalen begonnen worden waren.

Tabelle 2

Sexuelle Differenzierung „kleiner“ bzw. „großer" Glasaale

\begin{tabular}{|c|c|c|c|}
\hline \multicolumn{2}{|c|}{ Atlantische Aaale } & \multicolumn{2}{|c|}{ Mittelmeeraale } \\
\hline $\begin{array}{l}\text { Mittlere Länge bei } \\
\text { Versuchsbeginn }\end{array}$ & $\delta \delta: q 9$ & $\begin{array}{l}\text { Mittlere Länge bei } \\
\text { Versuchsbeginn }\end{array}$ & $\delta \hat{\partial}: q q$ \\
\hline $7,2 \mathrm{~cm}$ & $17: 4$ & $7,3 \mathrm{~cm}$ & $12: 2$ \\
\hline $7,7 \mathrm{~cm}$ & $9: 13$ & $8,9 \mathrm{~cm}$ & $22: 5$ \\
\hline
\end{tabular}


Die dargestellten Ergebnisse können die Behauptung von BeLliNi (1907), daß größere Glasaale vorwiegend zu Weibchen und kleinere Glasaale vorwiegend zu Männchen werden, zumindest für die atlantischen Aale stützen.

\section{Wachstumsleistung und Geschlecht}

Aus Abbildung 7 ist $z u$ ersehen, daß zahlenmäßig unter den Vorwüchsern die Männchen weitaus häufiger vertreten sind als die Weibchen, d. h. unter diesen Versuchsbedingungen sind die Männchen schneller gewachsen als die Weibchen.

Noch deutlicher wird diese Tatsache, wenn man die bei Versuchsende erreichte Durchschnittslänge der Männchen bzw. der Weibchen miteinander vergleidht. Atlantische Aale: $\bar{x} \mathrm{~L} \hat{\sigma}=32,2 \mathrm{~cm}(\mathrm{n}=28), \overline{\mathrm{x}} \mathrm{L} q=28,8 \mathrm{~cm}(\mathrm{n}=31)$; Aale aus dem Mittelmeer: $\bar{x} \mathrm{~L} \delta=34,3 \mathrm{~cm}(\mathrm{n}=34), \bar{x} \mathrm{~L} \rho=31,7 \mathrm{~cm}(\mathrm{n}=5)$. Diese Beobachtung machten schon andere Autoren bei der experimentellen Aufzucht von Aalen (Koops, MEske, mündl. Mitteilung). Sie steht im Gegensatz zu allen bisherigen Beobachtungen an natürlichen Aalbeständen.

\section{DISKUSSION}

Grundsätzlich scheint beim Aal - wie bei vielen anderen Fischen - die Geschlechtsbestimmung genetisch gesteuert zu sein. Für diese Annahme sprechen u. a. Untersuchungen des Karyotyps (Lieder, 1963; Chiarelli et al., 1969, Sick et al., 1962). Von den 19 Chromosomenpaaren, die bei Anguilla anguilla, A. rostrata und A. japonica vorhanden sind, ist ein Paar offensichtlich ungleich und wird als Gonosomenpaar gedeutet.

Neben den Ergebnissen anderer Autoren lassen die hier beschriebenen Experimente bei kritischem Rückblick jedoch erkennen, daß durch Überlagerung der genetischen Geschlechtsbestimmung des Aales durch Umwelteinflüsse eine modifikatorische Geschlechtsbestimmung nicht auszuschließen ist. Neben der Wirkung von Umweltfaktoren sprechen die vorliegenden Untersuchungen für eine mehr oder weniger starke genetische Determination bei verschiedenen geografischen Beständen. Die Fixierung des Geschlechtes erwies sich beispielsweise bei den Aalen aus dem Tyrrhenischen Meer als so stark, daß sie auch unter den offensichtlich die Entwicklung des weiblichen Ge-schlechts fördernden Bedingungen nodh zum Tragen kam. Ob diese Fixierung des Geschlechtes genetisch oder auf einem sehr frühen Stadium durch Umwelteinflüsse bedingt ist, läßt sich nicht $\mathrm{z}$ weifelsfrei beurteilen; die erste Annahme ist jedoch wahrscheinlicher.

Uber den Wirkungsgrad, mit dem die einzelnen Faktoren in dem komplizierten Zusammenspiel aller natürlichen Umweltbedingungen zum Tragen kommen, wissen wir zur Zeit noch weniger als über die Art der geschlechtsbestimmenden Faktoren selbst. Daher kann eine experimentelle Überprüfung der Rolle einzelner Faktoren 
immer nur für die jeweils im Versuch herrschende Kombination von Versuchsbedingungen gelten. Ein neu hinzutretender Faktor kann dementsprechend das ganze Ergebnis verändern. Hierin liegt möglicherweise eine Erklärung für die diesen Experimenten widersprechenden Ergebnisse von Fidora (1951) und D'ANCoNA (1957).

Nach allen bisherigen Befunden ist also für die Ausbildung des Geschlechtes beim Aal das Zusammenspiel zwischen genetischer Fixierung und ihrer Beeinflußbarkeit durch verschiedene Umweltfaktoren entscheidend. Dabei reicht die genetische Fixierung vom „stabilen" Männchen über einen breiten Bereich, in dem die genetische Fixierung des Geschlechtes mehr oder weniger labil ist, bis zum „stabilen" Weibchen. In dem Maße, wie die genetische Fixierung des Geschlechtes abnimmt, nimmt die Beeinflußbarkeit der sexuellen Differenzierung durch Umwelteinflüsse zu.

\section{ZUSAMMENFASSUNG}

1. An Glasaalen (Anguilla anguilla L.) aus dem Atlantik und dem Tyrrhenischen Meer wurde untersucht, inwieweit die Richtung der sexuellen Differenzierung von Haltungs- und Fütterungsbedingungen sowie von Größe und geografischer Herkunft der Aale abhängig ist bzw. beeinflußt werden kann.

2. Die histologischen Veränderungen während der Gonadenentwicklung werden beschrieben. Vor der definitiven geschlechtlichen Differenzierung wird ein intersexuelles Stadium durchlaufen.

3. Eine Beziehung der Geschlechtsdetermination zur geografischen Herkunft der Ver suchstiere wird - zumindest für die Glasaale aus dem Tyrrhenischen Meer - vermutet.

4. Ein gewisser Einfluß der Futterqualität konnte dahingehend festgestellt werden, daß nach Verfütterung von Kabeljau-Rogen eine leichte Verschiebung des Geschlechterverhältnisses zugunsten der 우 beobachtet wurde.

5. Die Aufzucht der Glasaale bei $17^{\circ}, 20^{\circ}, 23^{\circ}, 26^{\circ}$ und $29^{\circ} \mathrm{C}$ ergab, daß bei $26^{\circ} \mathrm{C}$ (optimale Wachstumstemperatur) ein zugunsten der $q \circ$ verschobenes Geschlechterverhältnis zutage tritt, während bei den anderen Temperaturen keine eindeutige Korrelation zur Temperatur verzeichnet werden konnte.

6. Die von Fidora (1951) bei der Teichaufzucht von Aalen gemachte Beobachtung, daß mit zunehmender Besatzdichte der Anteil an $\hat{\partial} \hat{\delta}$ ansteigt, konnte für diese Aquarienexperimente nicht bestätigt werden, denn trotz $200 \mathrm{fach}$ dichteren Besatzes waren bei Versuchsende die 우 in der Uberzahl.

7. Die Meinung von Bellini (1907), daß sich große Glasaale vorwiegend $\mathrm{zu}$ q$\propto$ und kleinere in erster Linie zu $\hat{\delta} \hat{\delta}$ entwickeln, wird durch die vorliegenden Ergebnisse für die atlantischen Aale gestützt.

8. Die $\delta$ zeigten unter allen Versuchsbedingungen ein rascheres Wachstum als die 우.

9. Der Zeitpunkt der sexuellen Differenzierung ist - unabhängig von Herkunft, Wassertemperatur und Fütterung - nicht vom Alter, sondern allein vom Erreichen eines bestimmten Längenbereiches (15-25 cm Körperlänge) abhängig. 
10. Es wird vermutet, daß die Geschlechtsbestimmung bei Anguilla anguilla aus dem Zusammenspiel genetischer Faktoren und verschiedener Umwelteinflüsse resultiert.

\section{ZITIERTE LITERATUR}

Ancona, U. DE, 1943. Nuove ricerche sulla determinazione sessuale dell'anguilla (Teil 1). Archo Oceanogr. Limnol. 3, 159-269.

- 1957. Nuove ricerche sulla determinazione sessuale dell'anguilla (2. Teil). Archo Oceanogr. Limnol. 11, 69-111.

- 1958. Comparative biology of eels in the Adriatic and in the Baltic. Verh. int. Verein. theor. angew. Limnol. 13, 731-735.

- 1959. Distribution of the sexes and environmental influence in the European eel. Archs. Anat. microsc. Morph. exp. 48 (Suppl.), 61-70.

BeCCARI, N, 1932. A proposito di nomenclatura nello studio delle cellule genitali degli Anfibi. Monitere zool. ital. 42 (Suppl.), 89.

Beldini, A., 1907. Expériences sur l'élevage de l'anguille en stabulation a Comacchio. Bull. Soc. cent. Aquic. Pêche 19, 81-93; 141-146; 186-196; 215-224.

Bertin, L., 1951. Les anguilles. Payot, Paris, 191 pp.

Chiarelli, B., 1969. The caryotype of some Teleostei fish obtained by tissue culture in vitro. Experientia 25, 426-427.

Frnora, M., 1951. Influenza dei fattori ambientali sull'accrescimento e sul differenziamento sessuale delle anguilla. Nova Thalassia 1, 3-37.

Frost, E. W., 1950. The eel fisheries of the River Bann, Northern Ireland, and observations on the age of the silver eels. J. Cons. perm. int. Explor. Mer 16, 358-383.

Gandolfr, A., Hornyold, 1932. Observations sur les sexe de la petite anguille jaune de l'Étang de Thau. Bull. Soc. Océanogr. Fr. 12, 1196-1199.

- 1931. Le sexe de la petite anguille de repeuplement du Marais de la Grande Brière après un séjour de trois ans dans un aquarium de Muséum. Bull. Mus. natn Hist. nat., Paris 3 (Sér. 2), 423.

Grassi, B., 1919. Nuove ricerche sulla storia naturale delle anguille. Memorie R. Com. talassogr, ital. Nr. 67.

Heldt, G. \& Heldt, H., 1929. Etudes sur les civelles de Sidi - Daoud. Bull. statist. Océanogr. Salammbo 16, 1-23.

Kuhumann, H., 1974. Experimentelle Untersuchungen über den Einfluß von Wassertemperatur und qualitativem Futterangebot auf das Wachstum und die Geschlechtsdifferenzierung des europäischen Aales. Diss., Univ. Hamburg, 154 pp.

Lreder, U., 1963. Uber vermutliche Gonosomen bei Perca, Acerina und Anguilla (Vertebratae, Pisces). Biol. Zbl. 82, 297-302.

- 1969. Verfahren für die Herstellung eines Alleinfutters für die Ernährung der Aale. Patentschrift DWP $65875 \mathrm{Kl} .53 \mathrm{~g}$ 4/04 DDR.

Matsui, I., 1952. Studies on the morphology, ecology and pond-culture of the Japanese eel (Ang. japonica). J. Shimonoseki Coll. Fish. 3 (8), 1-16.

Mazza, F., 1932. Contributa alle conoscenza dello sviluppo degli organi genitali delle anguille macroftalme o di calata e delle gialloverdognole o microftalme. Boll. Pesca Piscic. Idrobiol. 8, 600 .

Penáz, M. \& Tesch, F. W., 1970. Geschlechtsverhältnis und Wachstum beim Aal an verschiedenen Lokalitäten von Nordsee und Elbe. Ber. dt. wiss. Kommn. Meeresforsch. 21, 290.

Rodolico, A., 1933. Differenziamento dei sessi ed ovospermatogenesi nell'anguilla. Publ. Stat. zool. Napoli 13, 180-278.

RomeIs, B., 1968. Mikroskopische Technik. Oldenbourg, München, 757 pp.

SICK, K. M., 1962. Haemoglobin pattern and chromosome number of American, European and Japanese eels (Anguilla). Nature, Lond. 193, 1001-1002. 
SinHA, V. R. P. \& JonEs, J. W., 1967. On the age and growth of the freshwater eel (Anguilla anguilla). J. Zool., Lond. 153, 99-117.

SivertSen, E., 1938. Undersøkelser over forholdet mellem spiss-og bredhodet ål og deres naering. Rep. Norw. Fishery mar. Invest. 5, 1-22.

TrsCh, J. J., 1928. On sex and growth investigations of the freshwater eel in Dutch waters. Mitt. dt. SeefischVer. 34, 225-227.

Anschrift des Autors: Dr. H. Kunlmann

Bundesforschungsanstalt für Fischerei

Institut fïr Küsten- und Binnenfischerei

D-2 Hamburg 50

Palmaille 9

Bundesrepublik Deutschland 